\title{
Near infrared light variations of chemically peculiar stars. The SrCrEu stars ${ }^{\star}$
}

\author{
F.A. Catalano ${ }^{1,3}$, F. Leone ${ }^{2,3}$, and R. Kroll ${ }^{4}$ \\ 1 Istituto di Astronomia, Città Universitaria, Viale A. Doria 6, I-95125 Catania, Italy \\ 2 Osservatorio Astrofisico di Catania, Città Universitaria, Viale A. Doria 6, I-95125 Catania, Italy \\ 3 CNR-GNA, Unità di ricerca di Catania, Città Universitaria, Viale A. Doria 6, I-95125 Catania, Italy \\ ${ }^{4}$ Instituto de Astrofisica de Canarias, 38200 La Laguna, Tenerife, Spain
}

Received August 1; accepted October 13, 1997

\begin{abstract}
Twenty magnetic Chemically Peculiar (CP2) stars of the $\mathrm{SrCrEu}$ subgroup mostly brighter than the 7.5 visual magnitude have been investigated in the near infrared at 1.25, 1.6 and $2.2 \mu$. The stars HD 3980, HD 24712, HD 49976, HD 83368, HD 96616, HD 98088, HD 118022, HD 125248, HD 148898, HD 203006, and HD 220825 have been found to be variable in the infrared with the same period as the visible light, spectrum, and magnetic field variations. HD 221760 is also variable with a period of 12.45 days, which has to be confirmed. The stars HD 72968, HD 111133, HD 126515, HD 153882, and HD 164258 do show some hint of variability, although the data are too few. Infrared variability has been detected for the first time in the stars HD 101065, and HD 206088, which have not yet been considered as variable. No variability has been detected for the star HD 137949 within a time scale of the order of ten days.
\end{abstract}

Key words: stars: chemically peculiar — stars: variables: other - infrared: stars

\section{Introduction}

Until recently it was generally believed that the properties that characterize Chemically Peculiar stars (or CP stars, according to Preston's 1974 scheme): peculiar abundances, magnetic fields, and so on, do not influence the longer wavelength part of the spectrum, since the present knowledge indicates the absence of significant flux redistribution and less important line blocking in this region than at shorter wavelengths (Muthsam \& Weiss 1978; Hensberge \& Van Rensbergen 1986). In fact, starting from the consideration that the spectral peculiarities of the CP stars make

\footnotetext{
Send offprint requests to: F.A. Catalano

* Based on observations collected at the European Southern Observatory, La Silla Chile.
}

rather unreliable classical indirect methods for determining effective temperatures, Shallis \& Blackwell (1979) used infrared fluxes as cornerstones of their method of integrated fluxes (Blackwell \& Shallis 1977; Blackwell et al. 1979) which determines simultaneously effective temperature and angular diameters of stars.

Kroll et al. (1987) could finally show that the near infrared fluxes and colors of CP stars, when compared to a black body, are normal, like that of early main sequence stars. IRAS data could even prove that the normality of IR fluxes is guaranteed to at least $25 \mu$ (Kroll 1987): only two CP4 stars showed flux excesses longward of $60 \mu$, showing cold circumstellar material, which is not uncommon among early B stars.

Moreover Leone \& Catalano (1991) have shown that the solar composition Kurucz model atmospheres, which are used to fit the CP stars spectra from $\lambda 5500$ to $\lambda 16500 \AA$, give a fair representation of the overall flux distribution, with the exception of the Balmer region, where CP stars appear generally brighter than normal stars, this excess being just a few percent of the total flux.

In spite of this normality of the infrared behavior, peculiar abundances and/or magnetic fields seem to affect the near infrared. In fact, Catalano et al. (1991, hereafter CKL) have shown that, out of the eight CP stars monitored throughout their rotational periods, at least six are variable in the near infrared, although the amplitudes shown are smaller than in the visible.

In order to test the validity of common idea that the infrared region is not affected by all those phenomena that characterize the ultraviolet and visible parts of CP2 stars spectra, we have started an observational campaign to search for infrared variability, also in order to understand better the origin of the light variability, which is one of the outstanding observational aspects of these stars.

In this paper we report the results concerning twenty $\mathrm{CP} 2$ stars of the subgroup showing overabundances of $\mathrm{Sr}$, $\mathrm{Cr}$, and/or Eu. 
Table 1. Program stars, comparison stars, and their characteristics. Spectral types for the program stars are taken from the General Catalogue of CP stars (Renson et al. 1991), those of comparison stars are from the Bright Star Catalogue (Hoffleit \& Jaschek 1982). IR magnitudes are from the present observations

\begin{tabular}{|c|c|c|c|c|c|c|c|c|c|c|c|c|c|}
\hline \multicolumn{7}{|c|}{ Program Stars } & \multicolumn{7}{|c|}{ Comparison Stars } \\
\hline $\mathrm{HD}$ & $\mathrm{HR}$ & Sp. type & $m_{V}$ & $<J>$ & $<H>$ & $<K>$ & HD & $\mathrm{HR}$ & Sp. type & $m_{V}$ & $<J>$ & $<H>$ & $<K>$ \\
\hline 3980 & 183 & $\mathrm{~A} 7 \mathrm{SrCrEu}$ & 5.71 & 5.606 & 5.621 & 5.598 & 4150 & 191 & A0 IV & 4.36 & 4.354 & 4.345 & 4.317 \\
\hline 24712 & 1217 & A9 $\mathrm{Sr}$ & 5.99 & 5.454 & 5.325 & 5.285 & 25165 & 1235 & gK5 & 5.90 & 3.109 & 2.340 & 2.159 \\
\hline 49976 & 2534 & $\mathrm{~A} 1 \mathrm{SrCrEu}$ & 6.29 & 6.301 & 6.333 & 6.321 & 49481 & - & B8 & 6.7 & 7.035 & 7.083 & 7.093 \\
\hline 72968 & 3398 & A2 $\mathrm{SrCr}$ & 5.72 & 5.717 & 5.754 & 5.752 & 73997 & 3285 & A8 IV & 6.15 & 6.640 & 6.666 & 6.657 \\
\hline 83368 & 3831 & $\mathrm{~A} 7 \mathrm{SrCrEu}$ & 6.17 & 5.750 & 5.647 & 5.614 & 82578 & - & A9 IV/V & 6.54 & 6.060 & 5.950 & 5.915 \\
\hline 96616 & 4327 & $\mathrm{~A} 3 \mathrm{Sr}$ & 5.15 & 5.066 & 5.060 & 5.049 & 95370 & 4293 & A3 IV & 4.39 & 4.155 & 4.104 & 4.075 \\
\hline 98088 & 4369 & A9 $\mathrm{SrCrEu}$ & 6.14 & 5.842 & 5.806 & 5.774 & 96620 & 4315 & Fo Vn & 6.09 & 5.531 & 5.393 & 5.347 \\
\hline 101065 & - & F0 HoDy & 8.02 & 7.166 & 7.001 & 6.955 & 101388 & - & A5 & 7.8 & 7.396 & 7.311 & 7.272 \\
\hline 111133 & 4854 & $\mathrm{~A} 1 \mathrm{SrCrEu}$ & 6.34 & 6.341 & 6.376 & 6.368 & 109860 & 4805 & $\mathrm{~A} 1 \mathrm{~V}$ & 6.33 & 6.288 & 6.301 & 6.282 \\
\hline 118022 & 5105 & A2 $\mathrm{CrEuSr}$ & 4.94 & 4.915 & 4.940 & 4.926 & 121607 & 5244 & $\mathrm{~A} 8 \mathrm{~V}$ & 5.91 & 5.507 & 5.402 & 5.359 \\
\hline 125248 & 5355 & $\mathrm{~A} 1 \mathrm{EuCr}$ & 5.87 & 5.902 & 5.922 & 5.917 & 124683 & 5332 & $\mathrm{~A} 1 \mathrm{~V}$ & 5.43 & 5.582 & 5.606 & 5.599 \\
\hline 126515 & - & A2 $\mathrm{CrSr}$ & 7.1 & 7.132 & 7.164 & 7.162 & 121607 & 5244 & $\mathrm{~A} 8 \mathrm{~V}$ & 5.91 & 5.515 & 5.399 & 5.367 \\
\hline 137949 & - & F0 SrEuCr & 6.7 & 6.352 & 6.319 & 6.293 & 138268 & 5756 & A8 $\mathrm{V}$ & 6.22 & 5.938 & 5.857 & 5.824 \\
\hline 148898 & 6153 & $\mathrm{~A} 7 \mathrm{Sr}$ & 4.45 & 4.261 & 4.233 & 4.210 & 150453 & 6202 & F4 IV & 5.57 & 4.718 & 4.478 & 4.425 \\
\hline 153882 & 6326 & $\mathrm{~A} 1 \mathrm{CrEu}$ & 6.31 & 6.227 & 6.205 & 6.178 & 153809 & - & A0 & 7.0 & 7.089 & 6.997 & 6.954 \\
\hline 164258 & 6709 & A3 $\mathrm{SrCrEu}$ & 6.37 & 6.069 & 6.019 & 5.982 & 164259 & 6710 & F2 IV & 4.62 & 3.955 & 3.772 & 3.725 \\
\hline 203006 & 8151 & A2 $\mathrm{CrEuSr}$ & 4.82 & 4.744 & 4.725 & 4.700 & 202135 & 8117 & K2 III & 6.21 & 5.885 & 5.924 & 5.915 \\
\hline 206088 & 8278 & F0 p & 3.68 & 3.202 & 3.093 & 3.045 & 206677 & 8302 & Fo V & 5.99 & 5.565 & 5.460 & 5.418 \\
\hline 220825 & 8911 & $\mathrm{~A} 1 \mathrm{CrSr}$ & 4.95 & 4.957 & 4.979 & 4.968 & 221675 & 8944 & $\mathrm{~A} 2 \mathrm{~m}$ & 5.87 & 4.609 & 4.065 & 3.948 \\
\hline 221760 & 8949 & $\mathrm{~A} 2 \mathrm{SrCrEu}$ & 4.70 & 4.547 & 4.525 & 4.498 & 220401 & 8896 & Ko & 6.09 & 3.681 & 2.932 & 2.753 \\
\hline
\end{tabular}

\section{Observations}

We mainly selected bright stars, i.e. not fainter than about 7.5 mag, whose period was known from visible light variability.

The observations have been carried out in the near IR bands $J, H$, and $K$ at the $1 \mathrm{~m}$ photometric telescope at ESO, La Silla, Chile, using an InSb detector cooled with liquid nitrogen. Central wavelengths and bandwidths (in $\mu \mathrm{m})$ of the used filters are:

$\begin{array}{lll}J & 1.24 & 0.32 \\ H & 1.63 & 0.28 \\ K & 2.19 & 0.39\end{array}$

A detailed description of the ESO infrared photometers can be found in Bouchet (1989). The integration times, the number of cycles, and the desired rms accuracy in the mean level were optimized to get a $2 \%$ maximum error in the observations: the resulting accuracy in the final reduced data is typically 0.006 mag. ESO standard software was used for all reduction steps.

All program stars were measured relative to closeby comparisons, which were chosen to have as similar color and brightness as possible. Magnitudes in the standard IR system (Bouchet et al. 1991) have also been obtained by observing suitable standard stars from the ESO list. The program stars and their comparisons are listed in Table 1.

The data have been collected during several observing runs from July 1986 through January 1993. The list of the observing runs is given in Table 2 together with the symbols used in the figures to identify the observations made in each run.

For many stars we could get phase covering observations which we shall present here in more detail. 
Table 2. The list of the runs and the symbols used to represent in the figures the observations collected in different runs

\begin{tabular}{|clcc|}
\hline Run N. & Dates & symbol & observer \\
\hline 1 & 1986 Oct. $13-23$ & $\square$ & RK \\
2 & 1987 Nov. $1-8$ & $\nabla$ & FAC \\
3 & 1989 Apr. $19-26$ & $\circ$ & FAC \\
4 & 1991 Mar. $23-31$ & $\bullet$ & FAC \\
5 & 1991 Oct. $19-31$ & $\times$ & FAC \\
6 & 1993 Jan. $19-31$ & $\square$ & FAC \\
7 & 1993 Oct. $1-11$ & $*$ & FAC \\
\hline
\end{tabular}

\section{Light curves of individual stars}

The assumed ephemeris elements of the infrared light curves for the programme stars are listed in Table 3; they have mainly been taken from Catalano \& Renson (1984, 1988, 1997), Catalano et al. (1991, 1993), and references therein.

For each light curve a least square fit of all the data has been performed with a function of the type:

$$
\begin{aligned}
\Delta m=A_{0} & +A_{1} \sin \left(2 \pi\left(t-t_{0}\right) / P+2 \pi \phi_{1}\right) \\
& +A_{2} \sin \left(4 \pi\left(t-t_{0}\right) / P+2 \pi \phi_{2}\right)
\end{aligned}
$$

In this relation $\Delta m$ is the magnitude difference in each filter between the $\mathrm{CP}$ star and the comparison star, $t$ is the JD date, $t_{0}$ is the assumed initial epoch, $P$ is the period in days. This procedure can be partially accounted for by considering that within the accuracy of the measurements a sine wave and its first harmonic appear to be generally adequate to describe the light curves (North 1984; Mathys \& Manfroid 1985) and the magnetic field variations (Borra \& Landstreet 1980; Bohlender et al. 1993).

In the figures, where the infrared variations are plotted, open squares do represent the data collected in the 1986 run (i.e. the data with an accuracy lower than 0.01 mag), while the other symbols, listed in Table 2, represent the observations of the successive runs, having a better accuracy. In the same figures, the continuous line represents the fit to the observations obtained by means of Eq. (1) and which has to be considered only as indicative, just to evidentiate the observed variations. For most of the stars the individual fit of the differential $J, H$, and $K$ variations did show very similar behavior although with different amplitudes.

Indeed in the fits performed by means of (1), whose $A_{1}$ and $A_{2}$ coefficients and $\sigma$ are given in the last three columns of Table 3 , the second harmonic was not retained for the stars HD 72968 and HD 126515, because of a too small number of observations (HD 72968) and of a very poor phase coverage (HD 126515).

HD $3980 A=H R 183 A=\xi$ Phe $A$

An analysis of the photometric and magnetic variability of the late type CP2 star HD 3980 has been carried out by Maitzen et al. (1980) who found the period to be 3.9516 $( \pm 0.0003)$ days. The visible light curves show a double wave with different amplitudes, the maximum amplitude occurring in the $v$ filter, where it amounts to $0.13 \mathrm{mag}$ peak to peak. A well defined double-wave variation with the same period is indeed shown by the peculiarity index $\Delta a$ (Maitzen \& Vogt 1983).

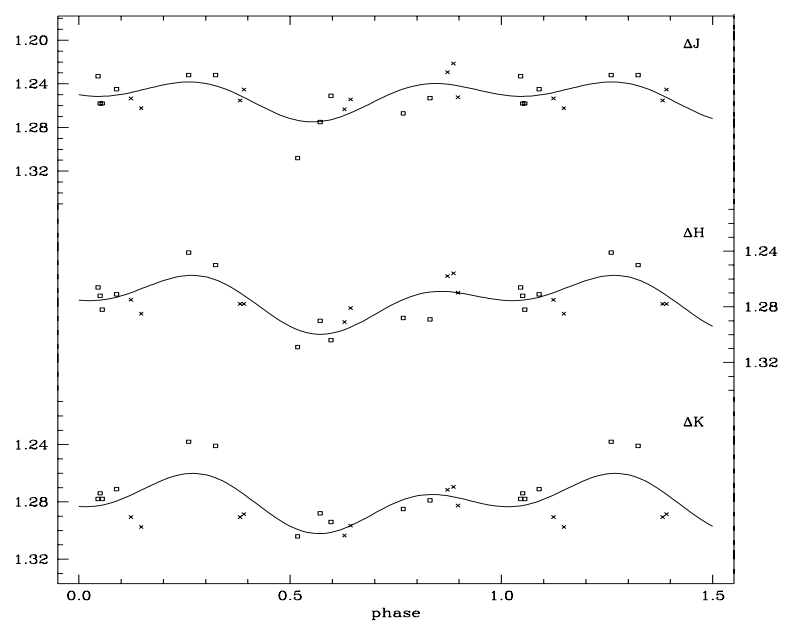

Fig. 1. Differential infrared light curves of HD 3980. The phases are computed according to the ephemeris elements in Table 3. The solid line is a least-square fit of the observations by Eq. (1) as described in the text

The infrared variability of HD 3980 was discovered by CKL. However further infrared observations have been carried out which are here reported. The infrared differential light curves, displayed in Fig. 1, are plotted versus the phase computed by means of Maitzen et al.'s ephemeris elements reported in Table 3. From Fig. 1 a double wave variation is quite evident with the same amplitude (of the order of 0.03 mag peak to peak) in all three filters, although it looks better defined in the $H$ and $K$ filters.

\section{$H D 24712=H R 1217=D O E r i$}

The cool magnetic star HD 24712 has had many studies made since the measurements of the magnetic field strength, of the radial velocity and of the line strengths of $\mathrm{Mg}$ and Eu carried out by Preston (1972), who found all of these to vary with a period of 12.448 days. 
Table 3. Ephemeris elements used to compute the phases of the variations, coefficients of the fits and their errors

\begin{tabular}{|c|c|c|c|c|c|c|c|}
\hline $\mathrm{HD}$ & $\mathrm{JD}(\phi=0)$ & Instant of & $P$ (days) & coeff. & $J$ & $H$ & $K$ \\
\hline 3980 & 2442314.48 & $v \min$. & 3.9516 & $\begin{array}{c}A_{1} \\
A_{2} \\
\sigma\end{array}$ & $\begin{array}{r}-0.0114 \\
0.0116 \\
0.0130\end{array}$ & $\begin{array}{r}-0.0138 \\
0.0125 \\
0.0105\end{array}$ & $\begin{array}{r}-0.0126 \\
0.0135 \\
0.0136\end{array}$ \\
\hline 24712 & 2440577.23 & $<H_{\mathrm{z}}>$ pos. extr. & 12.4610 & $\begin{array}{c}A_{1} \\
A_{2} \\
\sigma\end{array}$ & $\begin{array}{r}-0.0031 \\
-0.0095 \\
0.0093\end{array}$ & $\begin{array}{r}-0.0027 \\
0.0059 \\
0.0052\end{array}$ & $\begin{array}{r}0.0076 \\
-0.0077 \\
0.0058\end{array}$ \\
\hline 49976 & 2441615.10 & $u v b y \max$ & 2.97666 & $\begin{array}{c}A_{1} \\
A_{2} \\
\sigma\end{array}$ & $\begin{array}{l}0.0090 \\
0.0088 \\
0.0137\end{array}$ & $\begin{array}{l}0.0107 \\
0.0055 \\
0.0089\end{array}$ & $\begin{array}{l}0.0080 \\
0.0035 \\
0.0072\end{array}$ \\
\hline 72968 & 2432897.68 & $H_{\text {eff }} \max$. & 11.305 & $\begin{array}{c}A_{1} \\
A_{2} \\
\sigma\end{array}$ & $\begin{array}{c}-0.0073 \\
-\overline{0} \\
0.0047\end{array}$ & $\begin{array}{l}0.0002 \\
- \\
0.0037\end{array}$ & $\begin{array}{c}0.0069 \\
- \\
0.0033\end{array}$ \\
\hline 83368 & 2444576.169 & $<H_{\mathrm{z}}>$ neg. extr. & 2.851982 & $\begin{array}{c}A_{1} \\
A_{2} \\
\sigma\end{array}$ & $\begin{array}{l}0.0021 \\
0.0075 \\
0.0072\end{array}$ & $\begin{array}{r}-0.0021 \\
0.0043 \\
0.0069\end{array}$ & $\begin{array}{r}0.0017 \\
-0.0045 \\
0.0088\end{array}$ \\
\hline 96616 & 2444329.000 & uvby max. & 2.4394 & $\begin{array}{c}A_{1} \\
A_{2} \\
\sigma\end{array}$ & $\begin{array}{r}0.0122 \\
-0.0056 \\
0.0040\end{array}$ & $\begin{array}{r}0.0086 \\
-0.0064 \\
0.0032\end{array}$ & $\begin{array}{r}0.0098 \\
-0.0079 \\
0.0067\end{array}$ \\
\hline 98088 & 2434419.130 & periastron & 5.905130 & $\begin{array}{c}A_{1} \\
A_{2} \\
\sigma\end{array}$ & $\begin{array}{r}-0.0084 \\
-0.0136 \\
0.0109\end{array}$ & $\begin{array}{r}0.0054 \\
-0.0094 \\
0.0095\end{array}$ & $\begin{array}{r}-0.0100 \\
0.0107 \\
0.0124\end{array}$ \\
\hline 101065 & 2447640.652 & - & 7.593 & $\begin{array}{c}A_{1} \\
A_{2} \\
\sigma\end{array}$ & $\begin{array}{r}-0.0110 \\
0.0016 \\
0.0098\end{array}$ & $\begin{array}{r}0.0039 \\
-0.0029 \\
0.0089\end{array}$ & $\begin{array}{r}0.0033 \\
-0.0047 \\
0.0087\end{array}$ \\
\hline 111133 & 2448597.040 & uvby max. & 16.30720 & $\begin{array}{c}A_{1} \\
A_{2} \\
\sigma\end{array}$ & $\begin{array}{r}0.0035 \\
-0.0091 \\
0.0046\end{array}$ & $\begin{array}{l}0.0054 \\
0.0087 \\
0.0059\end{array}$ & $\begin{array}{r}0.0047 \\
-0.0069 \\
0.0050\end{array}$ \\
\hline 118022 & 2434816.90 & $y \max$. & 3.722084 & $\begin{array}{c}A_{1} \\
A_{2} \\
\sigma\end{array}$ & $\begin{array}{l}0.0125 \\
0.0064 \\
0.0065\end{array}$ & $\begin{array}{l}0.0096 \\
0.0049 \\
0.0094\end{array}$ & $\begin{array}{r}-0.0028 \\
-0.0025 \\
0.0074\end{array}$ \\
\hline 125248 & 2430143.07 & $u \max$ & 9.295710 & $\begin{array}{c}A_{1} \\
A_{2} \\
\sigma\end{array}$ & $\begin{array}{r}-0.0062 \\
-0.0110 \\
0.0062\end{array}$ & $\begin{array}{r}-0.0092 \\
0.0096 \\
0.0041\end{array}$ & $\begin{array}{l}0.0051 \\
0.0105 \\
0.0046\end{array}$ \\
\hline 126515 & 2437015.0 & $H_{\mathrm{S}} \max$. & 129.99 & $\begin{array}{c}A_{1} \\
A_{2} \\
\sigma\end{array}$ & $\begin{array}{l}0.0157 \\
- \\
0.0103\end{array}$ & $\begin{array}{c}0.0080 \\
- \\
0.0077\end{array}$ & $\begin{array}{c}-0.0137 \\
- \\
0.0063\end{array}$ \\
\hline 148898 & 2447645.0 & - & 0.7462 & $\begin{array}{c}A_{1} \\
A_{2} \\
\sigma\end{array}$ & $\begin{array}{l}0.0041 \\
0.0068 \\
0.0059\end{array}$ & $\begin{array}{r}0.0025 \\
-0.0026 \\
0.0049\end{array}$ & $\begin{array}{l}0.0017 \\
0.0049 \\
0.0056\end{array}$ \\
\hline 153882 & 2432752.730 & pos. crossover & 6.00890 & $\begin{array}{c}A_{1} \\
A_{2} \\
\sigma\end{array}$ & $\begin{array}{r}-0.0109 \\
0.0104 \\
0.0038\end{array}$ & $\begin{array}{r}-0.0101 \\
0.0140 \\
0.0133\end{array}$ & $\begin{array}{r}-0.0109 \\
-0.0155 \\
0.0069\end{array}$ \\
\hline 164258 & 2448339.77 & uvby min. & 0.829 & $\begin{array}{c}A_{1} \\
A_{2} \\
\sigma\end{array}$ & $\begin{array}{r}-0.0052 \\
-0.0058 \\
0.0073\end{array}$ & $\begin{array}{r}-0.0046 \\
-0.0053 \\
0.0048\end{array}$ & $\begin{array}{r}-0.0053 \\
-0.0032 \\
0.0056\end{array}$ \\
\hline 203006 & 2440345.32 & uvby I max. & 2.1224 & $\begin{array}{c}A_{1} \\
A_{2} \\
\sigma\end{array}$ & $\begin{array}{r}-0.0029 \\
0.0072 \\
0.0137\end{array}$ & $\begin{array}{r}0.0045 \\
-0.0141 \\
0.0111\end{array}$ & $\begin{array}{r}-0.0048 \\
-0.0106 \\
0.0095\end{array}$ \\
\hline 206088 & 2441615.0 & - & 2.78 & $\begin{array}{c}A_{1} \\
A_{2} \\
\sigma\end{array}$ & $\begin{array}{r}0.0094 \\
-0.0040 \\
0.0103\end{array}$ & $\begin{array}{l}0.0054 \\
0.0025 \\
0.0099\end{array}$ & $\begin{array}{r}0.0022 \\
-0.0039 \\
0.0053\end{array}$ \\
\hline 220825 & 2446013.0 & & 1.418 & $\begin{array}{c}A_{1} \\
A_{2} \\
\sigma\end{array}$ & $\begin{array}{r}-0.0061 \\
0.0024 \\
0.0063\end{array}$ & $\begin{array}{r}0.0038 \\
-0.0036 \\
0.0057\end{array}$ & $\begin{array}{r}0.0109 \\
-0.0005 \\
0.0082\end{array}$ \\
\hline 221760 & 2448300.0 & uvby max. & 12.45 & $\begin{array}{c}A_{1} \\
A_{2} \\
\sigma\end{array}$ & $\begin{array}{l}0.0098 \\
0.0032 \\
0.0048\end{array}$ & $\begin{array}{l}0.0075 \\
0.0019 \\
0.0065\end{array}$ & $\begin{array}{r}0.0052 \\
-0.0008 \\
0.0074\end{array}$ \\
\hline
\end{tabular}

HD 24712 has been extensively studied by Kurtz and coworkers, with the aim of investigating the short period oscillations of the cool magnetic SrCrEu stars which have been successfully interpreted as high-overtone p-modes with the pulsation axis aligned with the magnetic axis of the star which is itself oblique to the rotation axis (the oblique pulsator model: Kurtz 1982; Kurtz \& Marang 1987; Kurtz et al. 1992, and references therein).
Magnetic field observations have recently been carried out by Mathys (1991), Bagnulo et al. (1995) and Mathys \& Hubrig (1997). The ephemeris elements resulting from these observations are given in Table 3.

The infrared variability of HD 24712 was also discovered by CKL, however some more infrared observations have been carried out which are here reported. The infrared differential light curves are plotted in Fig. 2 versus the phase computed by means of the above cited 


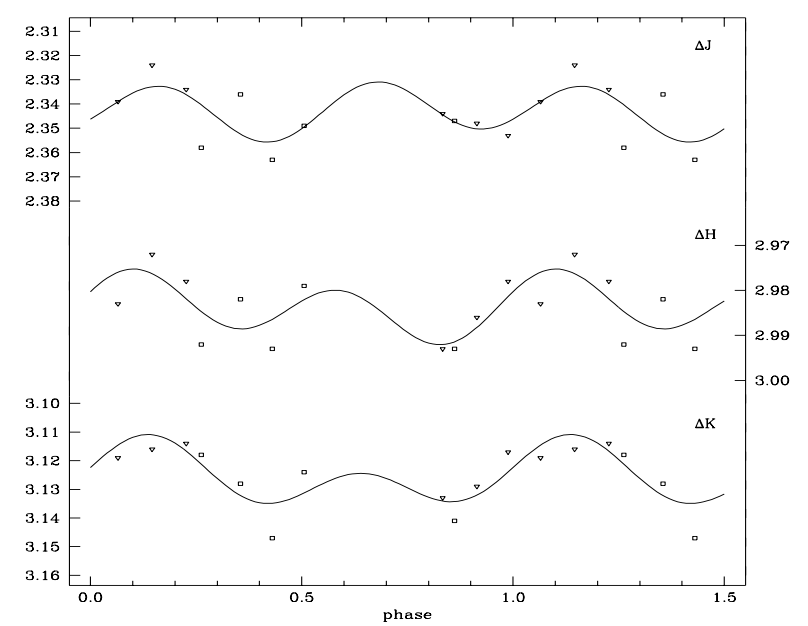

Fig. 2. Differential infrared light curves of HD 24712. The phases are computed according to the ephemeris elements in Table 3. The solid line is a least-square fit of the observations by Eq. (1) as described in the text

ephemeris elements. From this figure a double-wave variation in all three filters seems evident, with an amplitude of the order of 0.03 mag.

\section{HD $49976=H R 2534=$ V592 Mon}

A rather strong magnetic field was measured in HD 49976 by Babcock (1958a) who also found evidence of an extraordinary range of variation of the SrII lines.

Observations by Pilachowski et al. (1974), Maitzen \& Albrecht (1975), Mathys (1991), and Catalano \& Leone (1994) have allowed to refine the value of period to $2.97666 \pm 0.00008 \mathrm{~d}$.

The infrared differential light curves of HD 49976 are plotted in Fig. 3 versus the phase computed by means of the ephemeris elements reported in Table 3, where the assumed initial epoch is the actual time of maximum visible light as taken from the observations of Pilachowski et al. (1974). The infrared light variations are in phase with each other, and show quite the same amplitude, of the order of 0.03 mag peak to peak, in all three filters.

$$
\text { HD } 72968=H R 3398=3 \text { Hya }=H V H y a
$$

HD 72968 is the first star for which an estimate of the surface magnetic field intensity has been carried out on the basis of the study of the Zeeman intensification of spectral lines on the saturated part of the curve of growth (Hensberge \& de Loore 1974).

Photoelectric observations of HD 72968 have been carried out by Wolff \& Wolff (1971), Maitzen et al. (1978), Heck et al. (1987), and Catalano \& Leone (1990). From

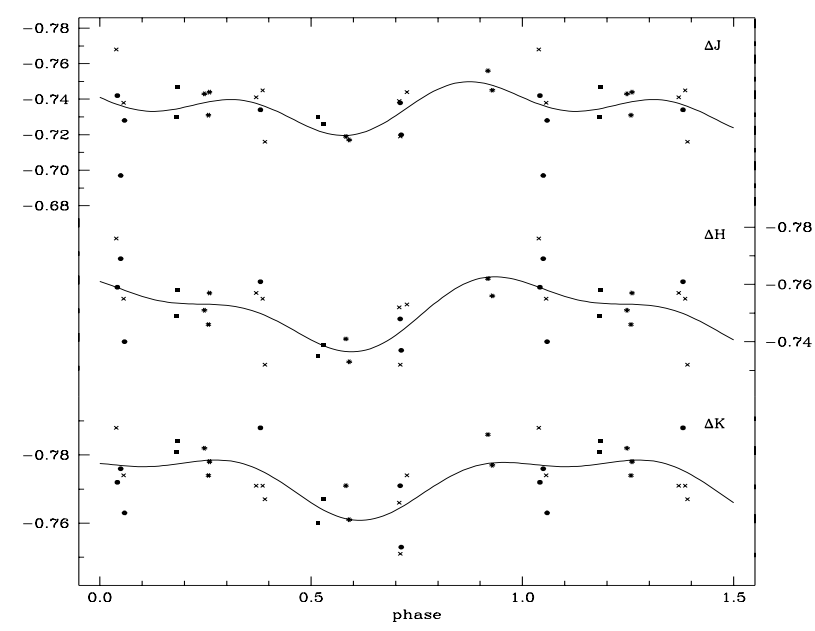

Fig. 3. Differential infrared light curves of HD 49976. The phases are computed according to the ephemeris elements in Table 3. The solid line is a least-square fit of the observations by Eq. (1) as described in the text

these studies the resulting value of the period of HD 72968 is $11.305 \mathrm{~d}$.

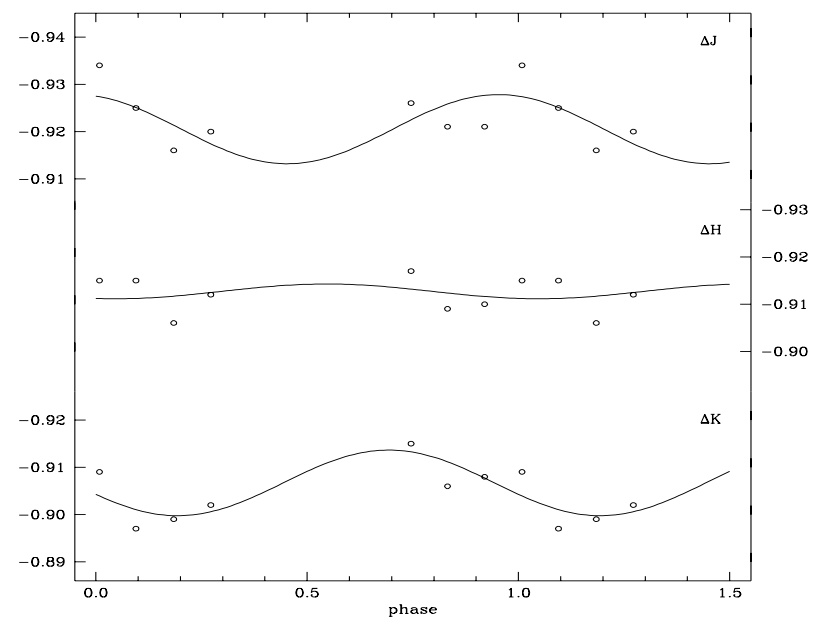

Fig. 4. Differential infrared light curves of HD 72968. The phases are computed according to the ephemeris elements in Table 3. The solid line is a least-square fit of the observations by Eq. (1) as described in the text

The infrared differential light curves of HD 72968 are plotted in Fig. 4 versus the phase computed on the basis of Maitzen et al. (1978) ephemeris elements reported in Table 3. From Fig. 4 a slight variability is better evident in the $K$ filter, with an amplitude of the order of 0.02 mag. 
$H D 83368=H R 3831=I M V e l$

The late type CP2 star HD 83368 is a visual double with a magnitude difference $\Delta V=2.85$ and a separation of 3.3 arcsec, which implies that both components are measured in the photometric observations.

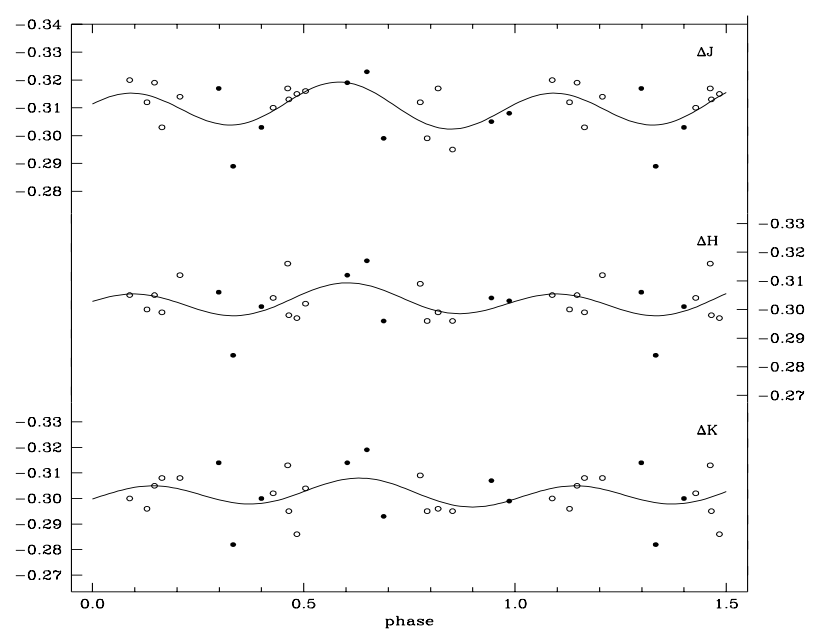

Fig. 5. Differential infrared light curves of HD 83368. The phases are computed according to the ephemeris elements in Table 3. The solid line is a least-square fit of the observations by Eq. (1) as described in the text

The photometric variability of HD 83368 has been detected by Renson \& Manfroid (1978). Thompson (1983) measured a magnetic field symmetrically variable from +800 to -800 gauss within a period of 2.857 d. Kurtz (1982) discovered that HD 83368 shows pulsations with periods of $\sim 6$ and 12 min modulated within a period of about 2.85 d. More recently Kurtz et al. (1992) have shown that the period of the pulsation amplitude modulation is equal to the period of the mean-light variation and have refined the rotation period to the value $2.851982 \pm 0.000005 \mathrm{~d}$. This value of the period has been confirmed by Mathys \& Manfroid (1985), Heck et al. (1987), Kurtz \& Marang (1988), and Catalano \& Leone (1994), and represents quite well also the magnetic observations of Mathys (1994) and Mathys \& Hubrig (1997).

The infrared differential light curves of HD 83368 are plotted in Fig. 5 versus the phase computed by means of Mathys \& Hubrig (1997) ephemeris elements reported in Table 3. From Fig. 5 we see that the HD 83368 shows a small amplitude double-wave variation, although with a quite large dispersion of the data, which could be due to the light dilution due to the presence of the close companion (Renson et al. 1984).

$$
\text { HD } 96616=H R 4327=4 G . C e n=V 815 C e n
$$

HD 96616 is a visual double with a secondary component 2.6 mag fainter than the primary and separated only by 2 arcsec.

The light variability of HD 96616 has been studied by Renson \& Manfroid (1977, 1978), Manfroid \& Renson (1983). However Manfroid \& Mathys (1985) noted an ambiguity between various aliases, preferring the value of $2.4394 \mathrm{~d}$.

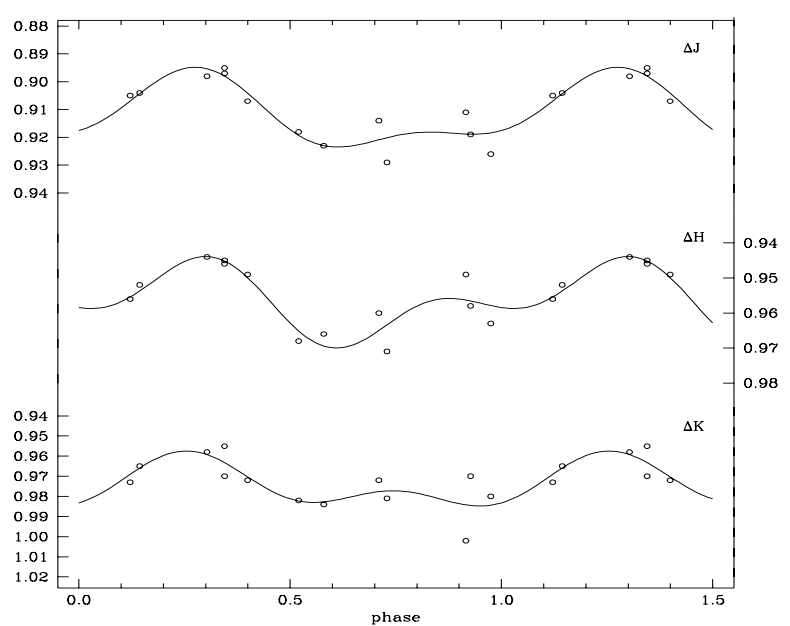

Fig. 6. Differential infrared light curves of HD 96616. The phases are computed according to the ephemeris elements in Table 3. The solid line is a least-square fit of the observations by Eq. (1) as described in the text

Only one measurement of the magnetic field of HD 96616 has been made by Borra \& Landstreet (1975) but no field significantly different from zero was measured.

Our infrared differential observations of HD 96616 are plotted in Fig. 6 versus the phase computed by means of the ephemeris elements reported in Table 3 . The infrared variation of HD 96616 is clearly evident in all three filters and amounts to about 0.03 mag peak to peak.

HD $98088=H R 4369=S V C r t$

HD 98088 is the brightest component of a visual binary (ADS 8115) whose components are separated by 57.2 arcsec and the secondary is 3.8 mag fainter. It is also a double-lined spectroscopic binary with a known orbit (Abt 1953; Abt et al. 1968; Wolff 1974) and a period of $5.90513 \mathrm{~d}$.

The magnetic field of HD 98088 has been measured by Babcock (1958a,b), who showed it to vary with the same period as the orbital motion.

The light variations of HD 98088 have been studied by Maitzen (1973), Wolff \& Morrison (1975), and Catalano 
\& Leone (1994). From all these data it appears that the period of this star is very well defined and still adequately represents observations carried out many years apart.

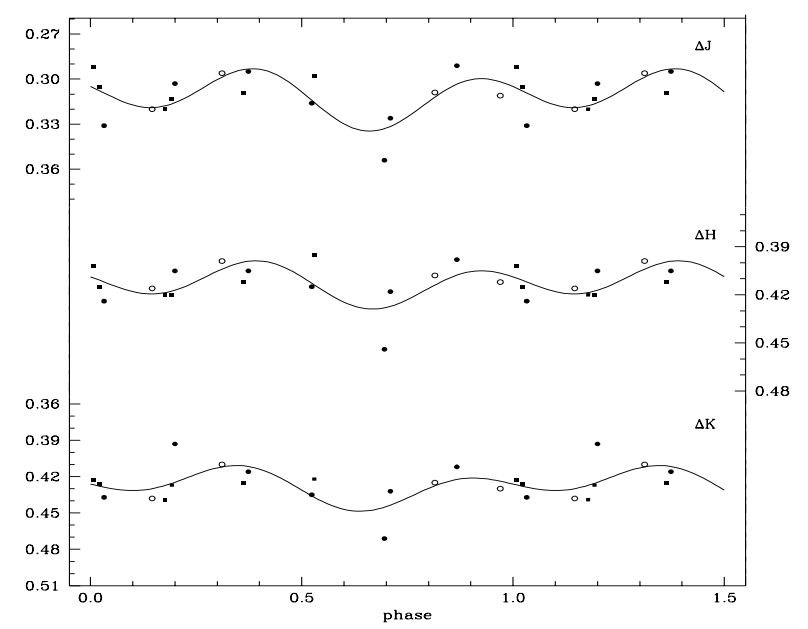

Fig. 7. Differential infrared light curves of HD 98088. The phases are computed according to the ephemeris elements in Table 3. The solid line is a least-square fit of the observations by Eq. (1) as described in the text

The infrared differential observations of HD 98088 are plotted in Fig. 7 versus the phase computed by means of Abt et al. (1968) ephemeris elements reported in Table 3. The infrared light variation of HD 98088 is double-waved in all three filters, with amplitudes of the order of 0.03 mag.

\section{HD $101065=C o D-46^{\circ}$ 7232 $=$ V816 Cen}

HD 101065, also known as Przybylski's star, is one of the most peculiar stars known: its visible spectrum is strongly dominated by the rare earth lines (Przybylski 1961, 1963, 1966; Cowley et al. 1977) whose abundances are estimated as high as five orders of magnitude if compared to cosmic abundances (Wegner \& Petford 1974). The iron peak elements, formerly considered to be underabundant in the visible spectrum, have been found to be strongly represented in the ultraviolet region $1900-3200 \AA$ (Wegner et al. 1983).

HD 101065 is an extremely highly blanketed star, so much that no available model atmosphere can fit the entire energy distribution as deduced from low resolution IUE spectra and visible and infrared photometry (Wegner et al. 1983). This fact can explain the difficulty in obtaining a reliable value of the effective temperature: values as low as $6075 \pm 200 \mathrm{~K}$ have been proposed (Przybylski 1977a).

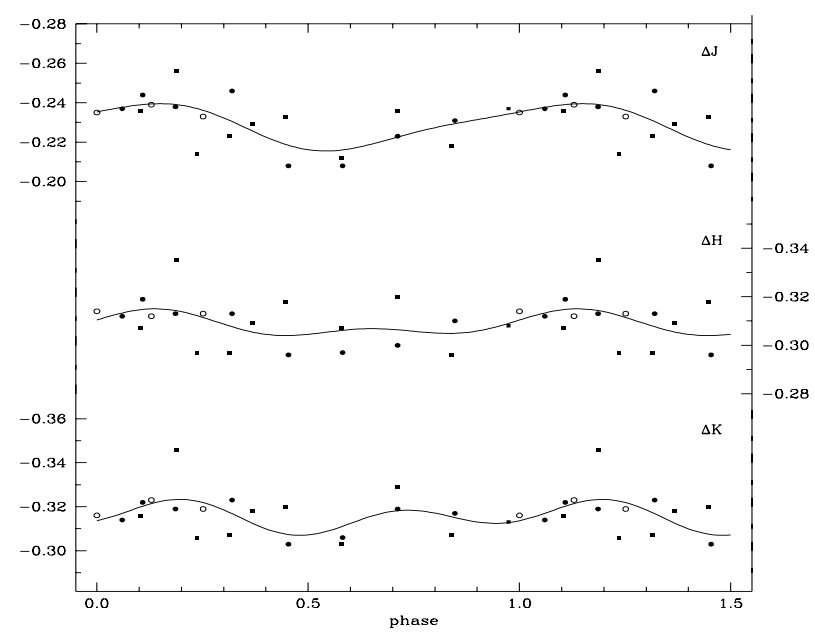

Fig. 8. Differential infrared light curves of HD 101065. The phases are computed according to the ephemeris elements in Table 3. The solid line is a least-square fit of the observations by Eq. (1) as described in the text

In spite of the several spectroscopic studies carried out, no clear evidence of variations of HD 101065 has been found so far in the line spectrum (Wolff \& Hagen 1976; Cowley et al. 1977) nor in the -2200 gauss magnetic field measured by Wolff \& Hagen (1976).

Photometric observations of HD 101065 aimed at detecting light variations have been carried out in the past by several authors. The most extensive photometric work has been done from 1969 to 1977 by Przybylski (1977b) who reported possible small amplitude long-period brightness variations of the order of two to three hundredths of a magnitude in Johnson $V$. However uvby observations appear to exclude systematic variations on a time scale of a week (Heck et al. 1976; Renson et al. 1976; Heck et al. 1987).

The only kind of variability so far detected to occur in HD 101065 is the rapid light variation with $P=$ 12.14 min. (Kurtz 1978; Kurtz \& Wegner 1979; Kurtz 1980, 1981). Kurtz (1982) discovered HD 101065 to show pulsations with periods of $\sim 12$ min modulated within a period of about $2.85 \mathrm{~d}$ and interpreted this result within the context of the oblique rotator model, suggesting the presence of a polarity reversing magnetic field.

Near infrared photometric observations have been carried out by several authors and some discrepancies are evident from these data (Hyland et al. 1975; Glass 1982). For this and on the basis of the fact that from Przybylski's (1977b) photometric observations some evidence of light variability could be present at the longer wavelengths, we decided to observe HD 101065 for variability in the near infrared. 
According to our observations spanning several runs, HD 101065 is quite clearly variable in the $J$ filter, although some hint of variability seems to be present in the other filters. Period search led to the value $7.596 \pm 0.005 \mathrm{~d}$, which was found to represent quite well the observations. Other possible period values are 0.858 and $1.827 \mathrm{~d}$, which however should be excluded if the rotational velocity $v_{e} \sin i$ is lower than $7 \mathrm{~km} \mathrm{~s}^{-1}$, as suggested by Wegner (1979) and Martinez \& Kurtz (1990).

The infrared differential observations of HD 101065 are plotted in Fig. 8 versus the phase computed by means of the ephemeris elements reported in Table 3.

\section{$H D 111133=H R 4854=E P$ Vir}

The presence of a fairly strong magnetic field in HD 111133 has been revealed by Babcock (1958a).

The magnetic variation has been studied by Wolff \& Wolff (1972), who found it occurring within a period of $16.31 \mathrm{~d}$. The same period was found for the spectral line intensity of CrI, CrII, FeI, and FeII, and the light variations. Further magnetic field measurements have been carried out by Glagolevsky et al. (1982).

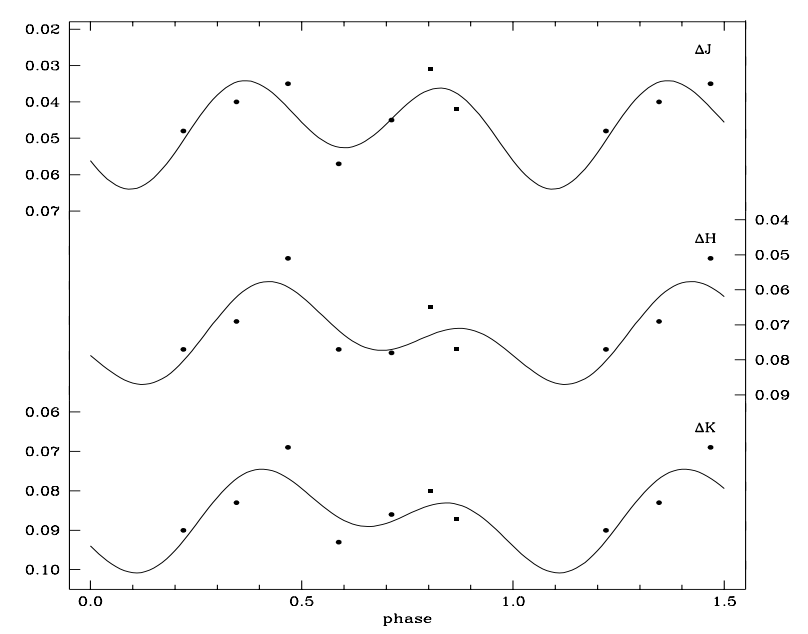

Fig. 9. Differential infrared light curves of HD 111133. The phases are computed according to the ephemeris elements in Table 3 . The solid line is a least-square fit of the observations by Eq. (1) as described in the text

The spectrum variations have been studied by Engin (1974), while the $\Delta a$ peculiarity index and $b-y$ variations have been studied by Buchholz \& Maitzen (1979).

Recent photometric observations of HD 111133 have been carried out by Adelman et al. (1992), Catalano \& Leone (1994) and North \& Adelman (1995). The best representation of all photometric observations was obtained by North \& Adelman (1995) whose ephemeris elements reported in Table 3, we have assumed in Fig. 9 to plot our infrared differential observations. From this figure it appears that the infrared light curves of HD 111133 show constant amplitudes of about 0.03 mag and are in phase with each other.

\section{$H D 118022=H R 5105=78$ Vir $=C W$ Vir}

HD 118022 is the first star in which a magnetic field has been detected (Babcock 1947). The magnetic field variation has been subsequently studied by Preston (1969), who first determined the correct period to be $3.7220 \mathrm{~d}$ from the analysis of the crossover effect. Several authors have provided further magnetic measurements (Wolff \& Wolff 1971; Wolff \& Bonsack 1972; Wolff 1978; Borra 1980; Borra \& Landstreet 1980; Leroy 1995), all of them confirming the period found by Preston.

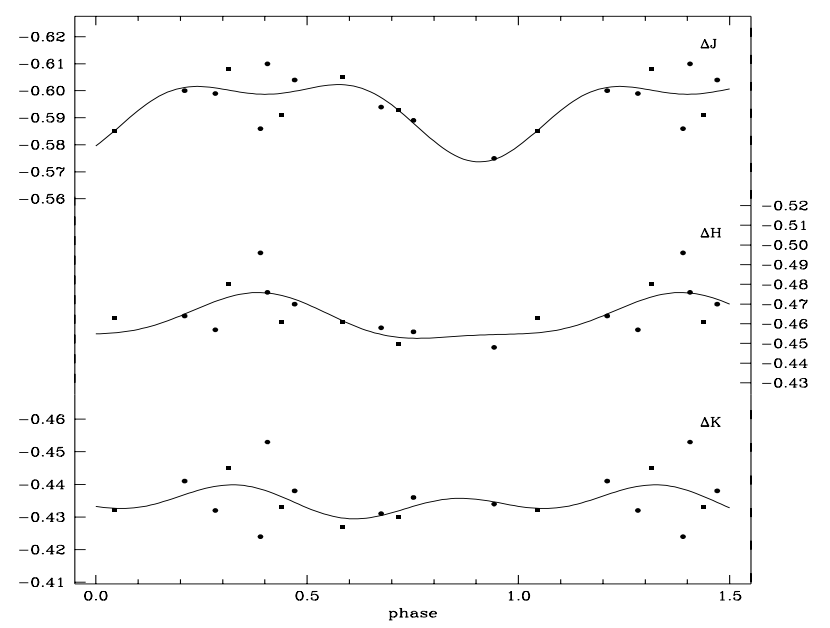

Fig. 10. Differential infrared light curves of HD 118022. The phases are computed according to the ephemeris elements in Table 3. The solid line is a least-square fit of the observations by Eq. (1) as described in the text

The light variability of HD 118022 has been studied by Stepien (1968), Winzer (1974), van Genderen (1971), Wolff \& Wolff (1971), and Catalano \& Leone (1994).

The infrared differential observations of HD 118022 are plotted in Fig. 10 with the ephemeris elements reported in Table 3. As it is evident from Fig. 10, HD 118022 is variable in $J$ and $H$, with light curves which are essentially single-waved and have amplitudes of about $0.04 \mathrm{mag}$, while it is almost constant in the $K$ filter.

\section{HD $125248=H R 5355=236 G$. Vir $=$ CS Vir}

HD 125248 is an outstanding magnetic, spectroscopic and light variable and is the first star for which an oblique rotator model has been put forward in order to describe the 
observed variations (Stibbs 1950). HD 125248 is also the first star for which Deutsch (1958) carried out a spherical harmonics analysis aimed at synthesizing a surface map of the abundance anomalies and of the magnetic field, based on his own spectroscopic observations (Deutsch 1947) and the magnetic field measurements of Babcock (1951). A lot of observational work has been devoted to HD 125248 (see Catalano \& Renson 1984, 1988, 1987, and Catalano et al. 1991, 1993 for references).

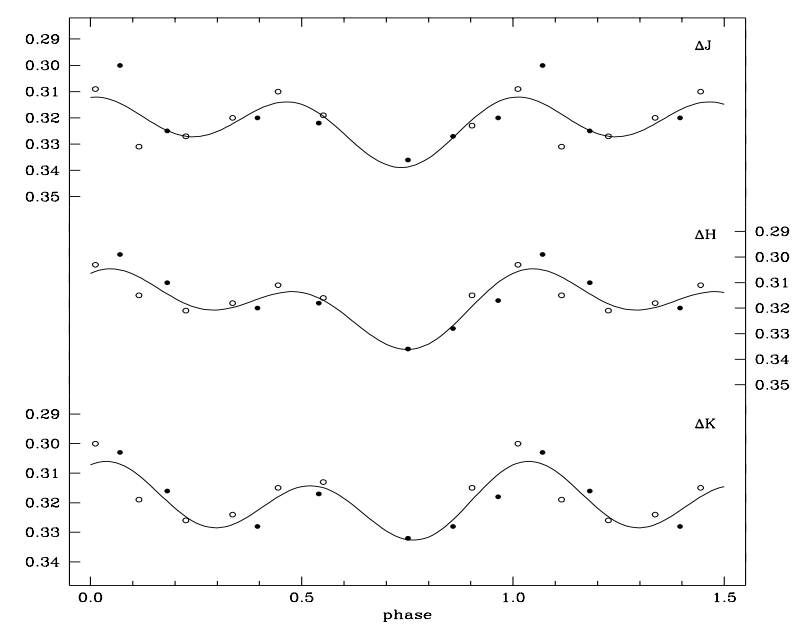

Fig. 11. Differential infrared light curves of HD 125248. The phases are computed according to the ephemeris elements in Table 3. The solid line is a least-square fit of the observations by Eq. (1) as described in the text

HD 125248 has been found to be variable in the near infrared by Catalano et al. (1992) with the same period as the visible light, spectrum and magnetic field variations. The infrared differential observations of HD 125248 are plotted in Fig. 11 versus the phase computed by means of the ephemeris elements reported in Table 3. The infrared variations do show a nearly constant amplitude larger than 0.03 mag peak-to-peak and look almost unchanged in all filters. All three light curves are in phase with each other, presenting a more pronounced double wave behavior than the visible light curves.

\section{HD $126515=G C 19462=F F$ Vir}

The star HD 126515 is the first star in which the spectral lines split in the Zeeman components have been observed. This fact allowed Preston (1970) to succeed in measuring the average surface field $H_{\mathrm{s}}$ which resulted to vary between 10 and $17 \mathrm{kG}$ with a periodicity of $130 \mathrm{~d}$. Preston also observed spectral line intensity variations of the lines of such elements as $\mathrm{Si}, \mathrm{Cr}, \mathrm{Fe}, \mathrm{Ti}, \mathrm{Sr}$, and $\mathrm{Eu}$ with the same period of the magnetic field and in phase with the magnetic variations.

From the $\Delta a$ study of the $\lambda 5200$ continuum depression carried out by Hensberge et al. (1986) it was inferred that the surface region, in which the high peculiarity values originate, are associated with the regions of enhanced spectral line strength, rather than with the local magnetic field.

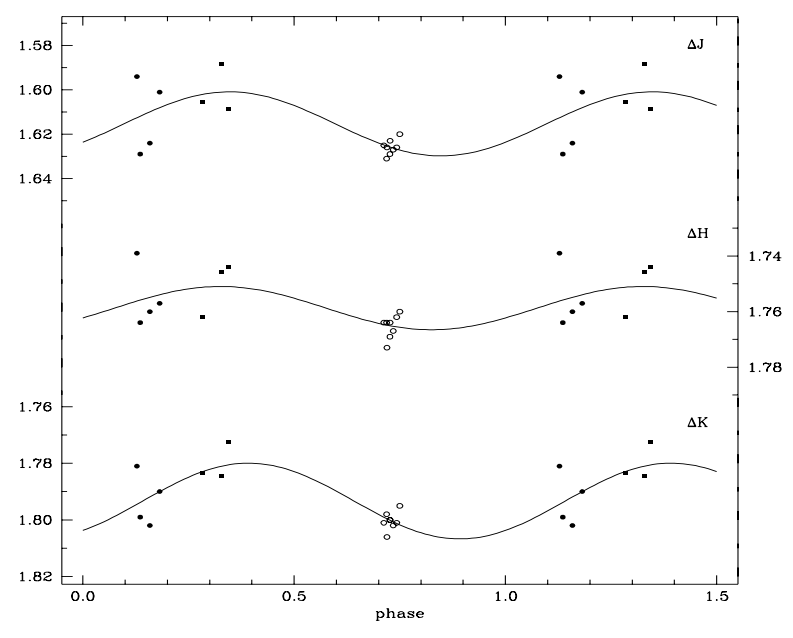

Fig. 12. Differential infrared light curves of HD 126515. The phases are computed according to the ephemeris elements in Table 3. The solid line is a least-square fit of the observations by Eq. (1) as described in the text

Photoelectric observations of HD 126515 have been carried out by Catalano \& Leone (1990) and North \& Adelman (1995). The latter authors refined the period to the value $129.99 \mathrm{~d}$. This value of the period has recently been confirmed by Mathys et al. (1997), from magnetic field observations.

The infrared differential observations of HD 126515 are plotted in Fig. 12 versus the phase computed by means of Mathys et al. (1997) ephemeris elements reported in Table 3. As it is evident from Fig. 12, due to the incomplete coverage of the light curves of HD 126515, nothing can be said about the amplitude of the variability.

$H D 137949=\zeta^{2} L i b=G Z L i b$

The unusual strength of the lines of SrII in the spectrum of HD 137949 has been noted by Adams et al. (1935). Babcock (1958a) also noted the unusual intensity of the EuII lines and found evidence of a rather strong magnetic field ( $\approx 1$ kgauss). Further magnetic measurements were carried out by van den Heuvel (1971), who suggested that the magnetic field variation occurs within $18.4 \mathrm{~d}$. This value of the period was not confirmed by Wolff (1975), 
who instead supported evidence of a magnetic variation occurring within a period of $23.26 \mathrm{~d}$, but did not detect light variations larger than 0.01 mag. Further magnetic observations have been carried out by Mathys et al. (1997), who have pointed out the possibility of a very long period ( $\geq 75$ years).

From photometric observations Kurtz (1982) suggested that the rotation period of HD 137949 could be 7.194 d. However, the light variability of this late CP star, if ever exists, is controversial. Essentially, no variability was evident from photometric observations carried out by Wolff (1975), Deul \& van Genderen (1983), and Catalano \& Leone (1994). No variations have also been found in the UV by van Dijk et al. (1978).

Our infrared observations are consistent with no light variation in excess of $0.01 \mathrm{mag}$.

\section{$H D 148898=H R 6153=\omega O p h$}

The spectrum variability of HD 148898 has been discovered by Morgan (1932) who noted that maxima and minima of the spectral line intensity of $\lambda 4215$ (SrII) occurred within a few days. Deutsch estimated the period to be of the order of $2 \mathrm{~d}$ (Bowen 1952). The existence of a polarity reversing magnetic field has been inferred by Babcock (1958a), but not measured because of the large spectral line width. Four measurements of the magnetic field intensity have been carried out by Borra \& Landstreet (1980) with low values inside the $3 \sigma$ level.

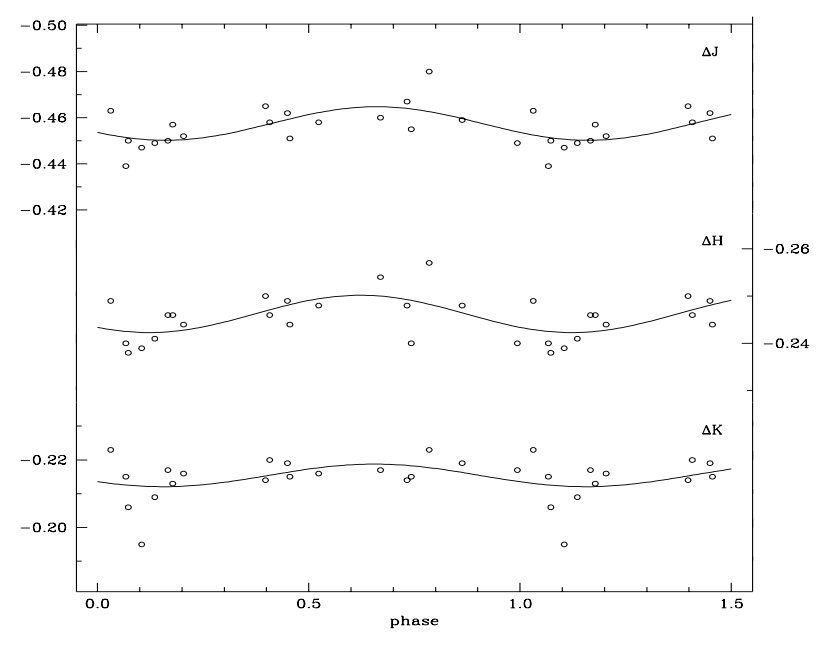

Fig. 13. Differential infrared light curves of HD 148898. The phases are computed according to the ephemeris elements in Table 3. The solid line is a least-square fit of the observations by Eq. (1) as described in the text

HD 148898 has been found to be a low amplitude light variable with several possible values of the period such as $0.7462 \pm 0.0002,1.4922 \pm 0.0004$, and 2.968 $\pm 0.003 \mathrm{~d}$ or nearby values (Renson \& Maitzen 1978). Subsequent uvby observations led to the most probable periodicities $1.79( \pm 0.02) \mathrm{d}, 4.67( \pm 0.08)$ d or, less probably, $2.33( \pm 0.02)$ d (Manfroid \& Mathys 1985; Mathys \& Manfroid 1985).

Among the above mentioned possible values, we have found that the best representation of our infrared differential observations was obtained with the period of $0.7462 \mathrm{~d}$, hence the infrared light curves of HD 148898 are plotted in Fig. 13 versus the phase computed by means of the ephemeris elements reported in Table 3. As it is evident from Fig. 13 the infrared variations of HD 148898 show an essentially single-waved trend and are in phase with each other, with very low amplitudes.

\section{$H D 153882=H R 6326=V 451 \mathrm{Her}$}

The star HD 153882 has been discovered by Gjellestad \& Babcock (1953) to be a magnetic variable with the period 6.005 d. Further magnetic observations of HD 153882 have been carried out by Hockey (1971), Preston \& Pyper (1965) and very recently by Mathys (1991) and Mathys \& Hubrig (1997).

The light variability of this star has been studied by Jarzebowski (1960), Chugainov (1961), Stepien (1968), van Genderen (1971), Panov \& Schöneich (1975), Schöneich et al. (1976), Rakosch \& Fiedler (1978), Hempelmann (1981), and Catalano \& Leone (1994). All these authors have confirmed the value $6.009 \mathrm{~d}$ of the period as given by Babcock (1958a).

Taking into account all magnetic data, relative to a time interval of more than forty years, Mathys (1991) refined the value of the period to $6.00890( \pm 0.000015) \mathrm{d}$. Mathys also confirmed the quite sinusoidal character with polarity reversal of the magnetic variation, occurring with the same period as the photometric one, and extrema in the range -1600 to +1600 gauss. Moreover Mathys studied the equivalent width of the FeII $\lambda 5961$ line and found large anharmonic variations whose extrema coincide in phase with the light variations but show no simple phase relation with the extrema of the magnetic field.

The infrared differential observations of HD 153882 are shown in Fig. 14, where they are plotted versus the phase computed by means of the ephemeris elements reported in Table 3. From Fig. 14 we see that HD 153882 is slightly variable in the infrared with an amplitude of the order of 0.04 mag peak to peak.

\section{HD $164258=H R 6709=$ V2126 Oph}

Babcock (1958a) included HD 164258 in the list of the stars with probable magnetic fields and noted the unusual strength of the SrII lines. Bonsack (1974) found indication of variability in the lines of EuII but did not determined any period. 


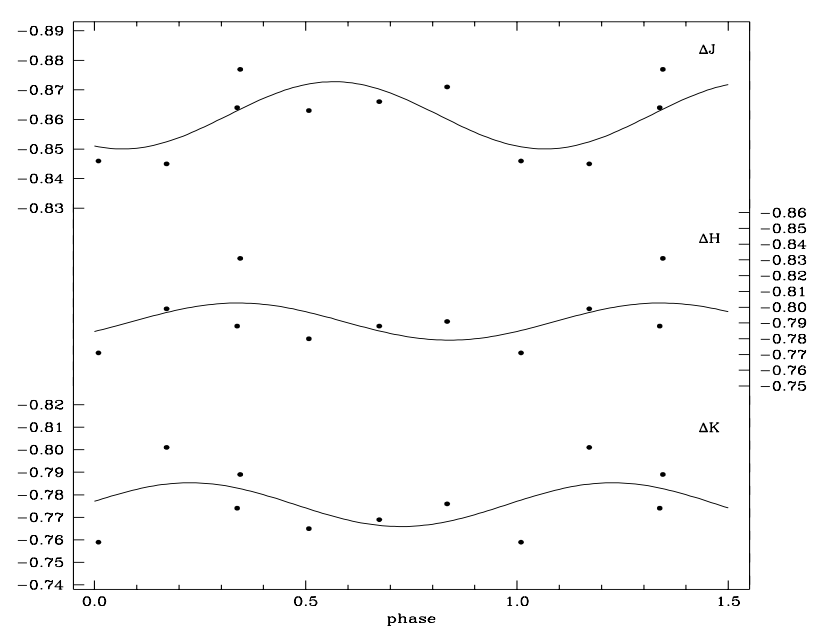

Fig. 14. Differential infrared light curves of HD 153882. The phases are computed according to the ephemeris elements in Table 3. The solid line is a least-square fit of the observations by Eq. (1) as described in the text

HD 164258 has been found to be variable by Renson \& Manfroid (1980) who gave indication of a period of about 2.41 d. However, Manfroid \& Mathys (1985) suggested shorter periods such as 0.719 or $0.359 \mathrm{~d}$. On the basis of new uvby observations Catalano \& Leone (1994) have found a period of $0.829( \pm 0.005) \mathrm{d}$, which gives quite a good representation of the observations.

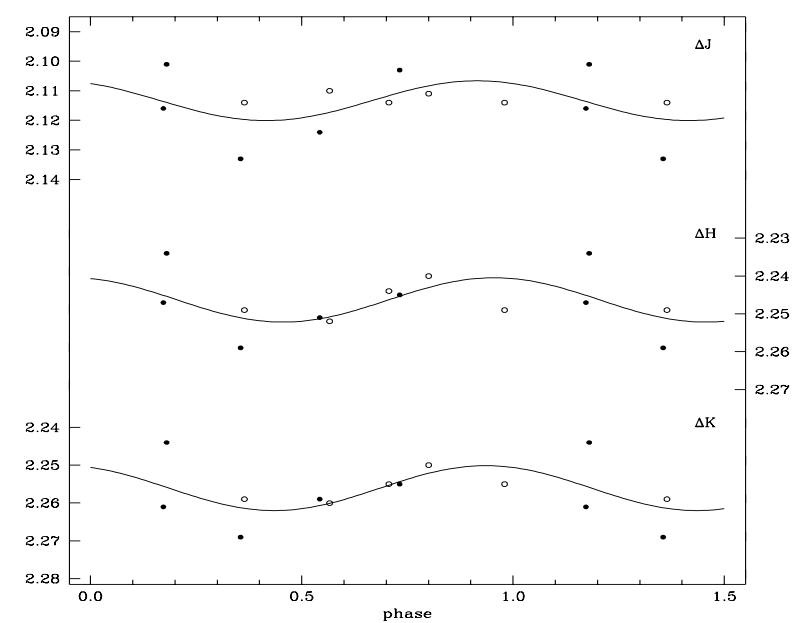

Fig. 15. Differential infrared light curves of HD 164250. The phases are computed according to the ephemeris elements in Table 3. The solid line is a least-square fit of the observations by Eq. (1) as described in the text
The infrared differential observations of HD 164258 are shown in Fig. 15, where they are plotted versus the phase computed by means of the ephemeris elements reported in Table 3. From Fig. 15 we see that HD 164258 shows a very small single-waved variation, better evident in $J$ where it has an amplitude of the order of $0.02 \mathrm{mag}$.

HD $203006=H R 8151=\theta^{1}$ Mic

HD 203006 has been found by Babcock (1958a) to be both spectrum and magnetic variable, on the basis of the fact that the magnetic field polarity appeared to reverse polarity in one day while lines of EuII and of SrII showed intensity variations of opposite sign.

The first determination of the period has been performed by Morrison \& Wolff (1971); from uvby observations these authors found HD 203006 to vary within $1.062 \pm 0.001 \mathrm{~d}$. Maitzen et al. (1974) found a double wave in their $U B V$ and uvby observations and stated the correct period to be $2.1219 \mathrm{~d}$. From more recent photoelectric observations Deul \& van Genderen (1983), have argued for a slightly shorter period, i.e. $2.1215 \pm 0.0001$, while from spectroscopic measurements Brandi \& Z̆ižn̆ovský (1990) favoured a slightly longer one, i.e. $2.1221 \pm 0.0002 \mathrm{~d}$, although both values are very near to Maitzen et al.'s value.

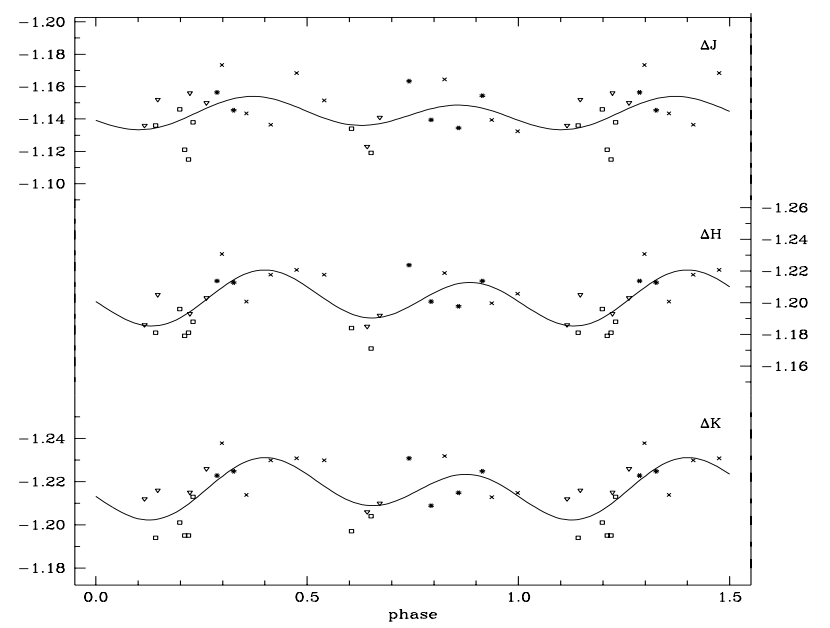

Fig. 16. Differential infrared light curves of HD 203006. The phases are computed according to the ephemeris elements in Table 3. The solid line is a least-square fit of the observations by Eq. (1) as described in the text

Our infrared differential observations of HD 203006 , phased with all of these period values, do show too large a dispersion, in fact they are better represented with a slightly longer period value, i.e. $2.1224 \mathrm{~d}$, which is just outside of the error bar. The resulting light curves are 
shown in Fig. 16, where they are plotted versus the phase computed by means of the ephemeris elements reported in Table 3. As it is evident from Fig. 16 the light curves of HD 203006 show essentially the same high dispersion double-waved variations, in phase with each other, as in the uvby, with amplitudes of about 0.04 mag.

$$
H D 206088=H R 8278=\gamma \text { Cap }
$$

HD 206088 was initially used as a standard, however, since we noted an anomalous scatter of the observations, it was included among the programme stars to look for variability.

The spectral type of HD 206088 is given as A8-F4 Sr in the General Catalogue of Ap and Am Stars (Renson et al. 1991), so it would be intermediate between late Ap stars and Am stars. No photometric nor spectroscopic studies of this star are available in the literature.

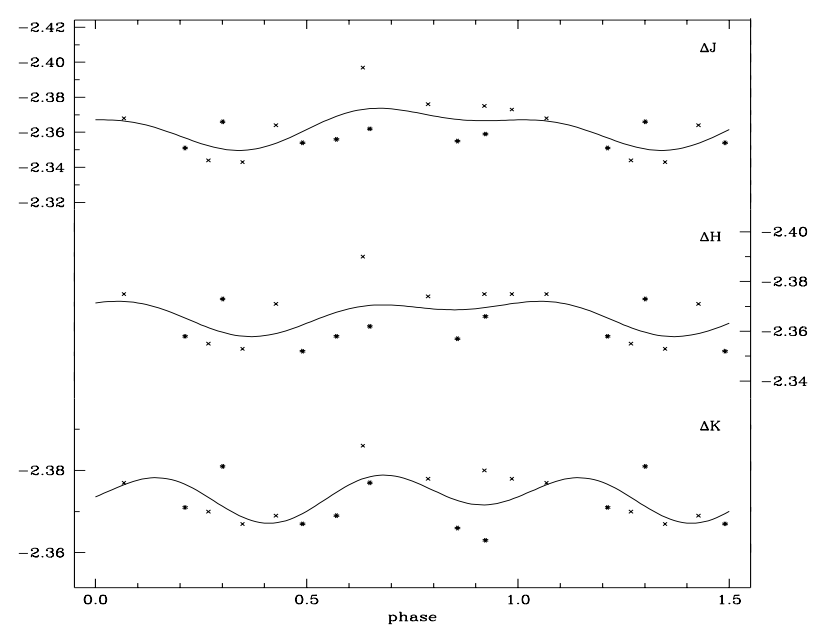

Fig. 17. Differential infrared light curves of HD 206088. The phases are computed according to the ephemeris elements in Table 3. The solid line is a least-square fit of the observations by Eq. (1) as described in the text

Period search led to the most probable value of the period of $2.78 \mathrm{~d}$, although many other nearby values are possible. However the number of the observed points is too small to allow a better determination. The infrared differential light curves of HD 206088 are shown in Fig. 17, where they are plotted versus the phase computed by means of the ephemeris elements reported in Table 3. As it is evident from Fig. 17, of HD 206088 might be variable, at least in the $J$ filter, but the period is too poorly defined to be fully reliable: it has to be considered as a trial value and needs to be confirmed.
$H D 220825=H R 8911=\kappa P s c$

A lot of photometric and spectroscopic studies of the SrCr star HD 220825 have been performed since Rakosch (1962) discovery of its variability with a period of $0.5805 \mathrm{~d}$. Recent studies have allowed to determine the period to be 1.418 d (Ryabchikova et al. 1996, and references therein). A small amplitude magnetic field variation (Borra \& Landstreet 1980) is also consistent with this period.

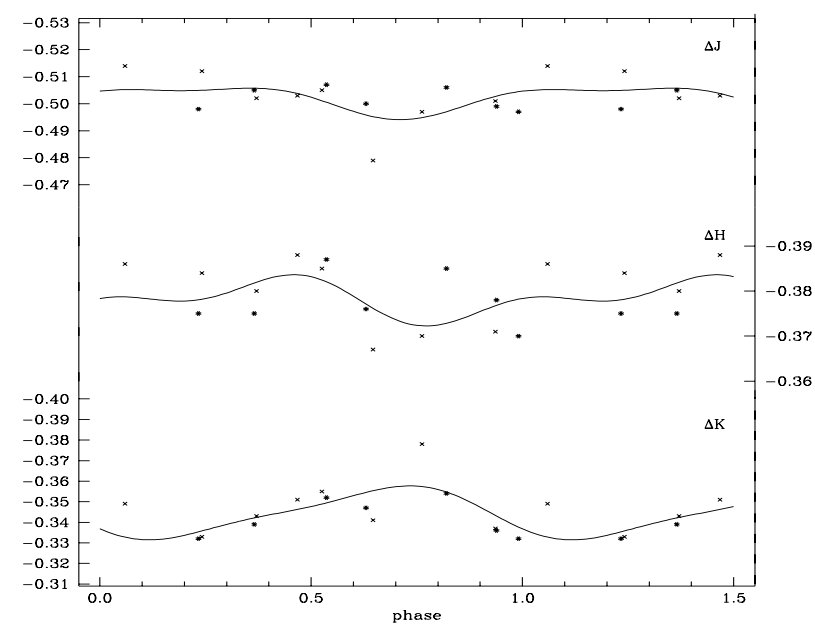

Fig. 18. Differential infrared light curves of HD 220825. The phases are computed according to the ephemeris elements in Table 3. The solid line is a least-square fit of the observations by Eq. (1) as described in the text

The infrared differential light curves of HD 220825 are shown in Fig. 18, where they are plotted versus the phase computed by means of the ephemeris elements reported in Table 3. As it is evident from Fig. 18, HD 220825 appears to be slightly variable in $K$ with an amplitude of the order of 0.03 mag.

\section{HD 221760 = HR $8949=\iota$ Phe}

Babcock (1958a) found the SrII, CrI and CrII lines to be particularly prominent in the spectrum of HD 221760 , and supported evidence for a rather weak magnetic field of positive polarity. Further magnetic field measurements by Borra \& Landstreet (1980) confirmed the weakness of the field $(\approx 0)$.

The only available photometric observations of this star are those by van Genderen (1971), who discovered HD 221760 to be variable in light with a period of $12.5 \mathrm{~d}$.

HD 221760 was found to be variable in the infrared, at least in the $J$ filter, and period search led to a number of values as 12.016, 12.224, 12.450, 12.655, and 12.886 days. We have preferred the value 12.45 days, which gives the minimum dispersion, but it has to be considered only 
as preliminary and should be confirmed. Our infrared differential observations of HD 221760 are plotted in Fig. 19 versus the phase computed by means of the ephemeris elements reported in Table 3. From Fig. 19 we see that HD 221760 might be variable in $J$ with an amplitude of the order of $0.02 \mathrm{mag}$. However, the period should be confirmed.

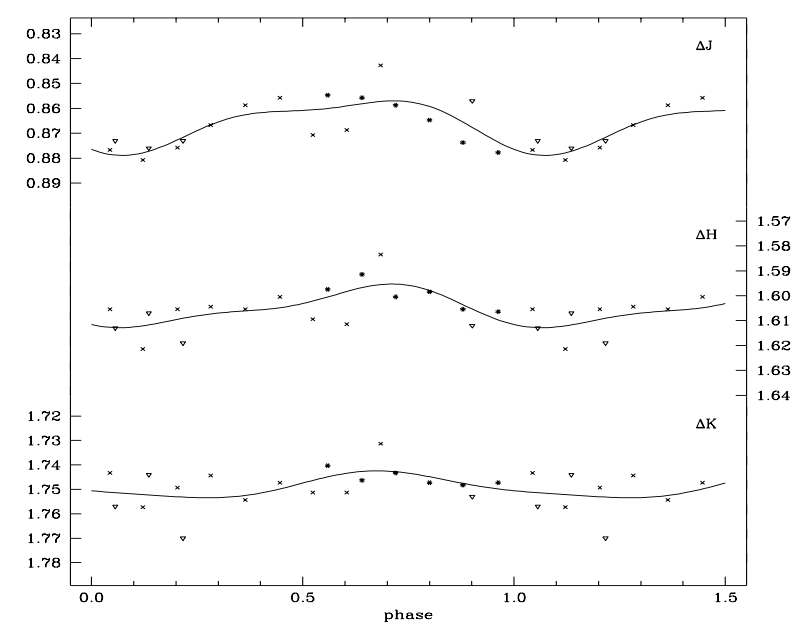

Fig. 19. Differential infrared light curves of HD 221760. The phases are computed according to the ephemeris elements in Table 3. The solid line is a least-square fit of the observations by Eq. (1) as described in the text

\section{Discussion and conclusions}

We have presented near infrared photometric observations of the CP2 SrCrEu stars HD 3980, HD 24712, HD 49976 , HD 72968, HD 83368, HD 96616, HD 98088, HD 101065, HD 111133, HD 118022, HD 125248, HD 126515, HD 137949, HD 148898, HD 153882, HD 164258, HD 203006, HD 206088, HD 220825, and HD 221760.

Infrared variability has been observed in all program stars but HD 137949, which has been found to be constant within 0.01 mag. Variability of the stars HD 101065 and HD 206088 has been detected for the first time, although their periodicity has to be confirmed.

From the analysis of the visible light variations we know that the amplitudes shown by $\mathrm{CP} 2$ of the $\mathrm{SrCrEu}$ subgroup are in the average smaller than those of hotter CP stars, but they show almost the same very complicated morphology of different behavior in different filters, in the sense that the shape of the variations and the phases at which their extrema occur do change with the wavelength. This result has to be taken into consideration when looking for the interpretation of the observed variations.
The typical trend of CP2 stars to present smaller amplitude light variations at increasing wavelength is confirmed: the amplitudes in the near infrared are smaller than in the visible. In most cases we find variations which appear to be in phase with each other in all filters.

The origin of light variations in the ultraviolet and visible part of the spectrum is still unclear, only qualitative considerations have been made based on the assumption that elements are not homogeneously distributed over the surface. Leckrone et al. (1974) and Leckrone (1976), pointing out that CP stars are flux deficient in the ultraviolet if compared to normal stars having the same Balmer jump, have suggested the presence of a greatly enhanced ultraviolet line opacity source, distributed more or less uniformly over the entire Balmer continuum region, and the redistribution of the absorbed UV flux longward of the null-wavelength region, that is the wavelength region with no observed variation. However the complex behavior of the visible light curves of some stars, as for example HD 125248, is a direct evidence that this mechanism cannot fully explain the observed light variations.

Another possible origin of the light variations is the local line blocking. However Pilachowski \& Bonsack (1975) have examined the influence of local line blocking on the light variations of HD 125248, and concluded that line blocking is certainly important but not sufficient to explain the observed amplitudes.

According to Babcock (1958a,b) and Deutsch (1958), Rare Earths and Fe are mainly concentrated in the positive magnetic pole region, while $\mathrm{Cr}$ and $\mathrm{Sr}$ are concentrated at the negative one. In a previous paper (CKL) we investigated the effects of high metallicity at the near infrared wavelengths. Because of the numerous metallic absorption lines, the blanketing mechanism steepens the temperature gradient and redistributes the flux from the ultraviolet, where the metallic absorption is strongest, to longer wavelengths, yet conserving the effective temperature. Since the atmospheres of CP stars show locally inhomogeneous metal distributions, the stellar rotation should communicate these optical depth variations as infrared variability. CKL have shown that a Kurucz model atmosphere with a metal content ten times the solar one could explain a three percent variation in the near infrared brightness, which is the typically observed value.

The possibility that the magnetic pressure could have non-negligible influence on the hydrostatic equilibrium of the stellar atmosphere has been suggested in the past to explain the light variations of CP stars, although it does not always match the observations. The characteristics of the observed infrared light variations could be considered supporting the magnetic pressure influence on the hydrostatic equilibrium pressure since the infrared radiation comes from the outermost layers where the gas pressure becomes lower. However, because of the overabundances in the magnetic pole regions, the expected modification of the atmospheric temperature gradient produced by the 
overabundant elements could be the origin of the infrared variations.

Acknowledgements. We would like to thank Dr. P. Bouchet and R. Vega Tello for their help and advice during the observations and reductions.

\section{References}

Abt H.A., 1953, PASP 65, 274

Abt H.A., Conti P.S., Deutsch A.J., Wallerstein G., 1968, ApJ 153,177

Adams W.S., Joy A.H., Humason M.L., Brayton A.M., 1935, ApJ 81, 187

Adelman S.J., Dukes R.J. Jr., Pyper D.M., 1992, AJ 104, 314

Babcock H.W., 1947, ApJ 105, 105

Babcock H.W., 1951, ApJ 114, 1

Babcock H.W., 1958a, ApJS 3, 141

Babcock H.W., 1958b, ApJ 128, 228

Bagnulo S., Landi degl'Innocenti E., Landolfi M., Leroj J.L., 1995, A\&A 295, 459

Blackwell D.E., Shallis M.J., 1977, MNRAS 180, 177

Blackwell D.E., Shallis M.J., Selby M.J., 1979, MNRAS 188, 847

Bohlender D.A., Landstreet J.D., Thompson I.B., 1993, A\&A 269, 355

Bonsack W.K., 1974, PASP 86, 408

Borra E.F., 1980, ApJ 235, 911

Borra E.F., Landstreet J.D., 1975, PASP 87, 961

Borra E.F., Landstreet J.D., 1980, ApJS 42, 421

Bouchet P., 1989, Infrared Photometry, E.S.O. Operating Manual No. 11

Bouchet P., Manfroid J., Schmider F.-X., 1991, A\&AS 91, 409

Bowen I.S., 1952, Mount Wilson and Palomar Observatories Ann. Rep., Carnegie Inst. of Washington Yearbook 51, 11

Brandi E., Žižn̆ovský J., 1990, Mitt. Karl-Schwarzschild Obs. Tautenburg Nr. 129 , p. 9

Buchholz M., Maitzen H.M., 1979, A\&A 73, 222

Catalano F.A., Leone F., 1990, A\&AS 83, 491

Catalano F.A., Leone F., 1994, A\&AS 108, 595

Catalano F.A., Renson P., 1984, A\&AS 55, 371

Catalano F.A., Renson P., 1988, A\&AS 72, 1

Catalano F.A., Renson P., 1997, A\&AS 121, 57

Catalano F.A., Kroll R., Leone F., 1991, A\&A 248, 179 (CKL)

Catalano F.A., Kroll R., Leone F., 1992, A\&A 263, 203

Catalano F.A., Renson P., Leone F., 1991, A\&AS 87, 59

Catalano F.A., Renson P., Leone F., 1993, A\&AS 98, 269

Chugainov P.F., 1961, Perem. Zvezdy 13, 255

Cowley C.R., Cowley A.P., Aikman G.C.L., Crosswhite H.M., 1977, ApJ 216, 37

Deul E.R., van Genderen A.M., 1983, A\&A 118, 289

Deutsch A.J., 1947, ApJ 105, 283

Deutsch A.J., 1958, Harmonic analysis of the periodic spectrum variables. In: Lehnert B. (ed.), Proc. I.A.U. Symp. 6, Electromagnetic Phenomena in Cosmical Physics. Cambridge University Press, Cambridge, p. 209

Gjellestad G., Babcock H.W., 1953, ApJ 117, 12

Glagolevskii Yu.V., Bychkov V.D., Iliev I.K., Romanyuk I.I., Chunakova N.M., 1982, Pis'ma Astron. Zh. 8, 26

Glass I.S., 1982, Mon. Not. Astron. Soc. South Africa 41, 117

Heck A., Manfroid J., Renson P., 1976, A\&AS 25, 143
Heck A., Mathys G., Manfroid J., 1987, A\&AS 70, 33

Hempelmann A., 1981, Astron. Nachr. 302, 47

Hensberge H., de Loore C., 1974, A\&A 37, 367

Hensberge H., Van Rensbergen W., 1986, in: Upper Main Sequence Stars with Anomalous Abundances, Cowley C.R. et al. (eds.) I.A.U. Coll. 90, p. 151

Hensberge H., Maitzen H.M., Catalano F.A., Schneider H., Pavlovski K., Weiss W.W., 1986, A\&A 155, 314

Hockey M.S., 1971, MNRAS 152, 97

Hoffleit D., Jaschek C., 1982, The Bright Star Catalogue, $4^{\text {th }}$ revised edition, Yale University Observatory

Hyland A.R., Mould J.R., Robinson G., Thomas J.A., 1975, PASP 87, 439

Jarzebowski T., 1960, Acta Astron. 10, 31

Kroll R., 1987, A\&A 181, 315

Kroll R., Schneider H., Catalano F.A., Voigt H.H., 1987, A\&AS 67, 195

Kurtz D.W., 1978, Inf. Bull. Var. Stars 1436

Kurtz D.W., 1980, MNRAS 191, 115

Kurtz D.W., 1981, MNRAS 196, 61

Kurtz D.W., 1982, MNRAS 200, 807

Kurtz D.W., Marang F., 1987, MNRAS 229, 285

Kurtz D.W., Marang F., 1988, MNRAS 231, 1039

Kurtz D.W., Wegner G., 1979, ApJ 232, 510

Kurtz D.W., Kanaan A., Martinez P., Tripe P., 1992, MNRAS 255,289

Leckrone D.S., 1976, Properties of Ap stars in the ultraviolet. In: Weiss W.W., Jenkner H., Wood H.J. (eds.) Proc I.A.U. Coll. 32, Physics of Ap Stars, Universitätssternwarte Wien, p. 465

Leckrone D.S., Fowler J.W., Adelman S.J., 1974, A\&A 32, 237

Leone F., Catalano F.A., 1991, A\&A 242, 199

Leroy J.C., 1995, A\&AS 114, 79

Maitzen H.M., 1973, A\&AS 11, 327

Maitzen H.M., Albrecht R., 1975, A\&A 44, 405

Maitzen H.M., Vogt N., 1983, A\&A 123, 48

Maitzen H.M., Albrecht R., Heck A., 1978, A\&A 62, 199

Maitzen H.M., Breysacher J., Garnier R., Sterken C., Vogt N., 1974, A\&A 32, 21

Maitzen H.M., Weiss W.W., Wood H.J., 1980, A\&A 81, 323

Manfroid J., Mathys G., 1985, A\&AS 59, 429

Manfroid J., Renson P., 1983, A\&AS 51, 267

Martinez P., Kurtz D.W., 1990, MNRAS 242, 636

Mathys G., 1991, A\&AS 89, 121

Mathys G., 1994, A\&AS 108, 547

Mathys G., Hubrig S., 1997, A\&A (in press, ESO Sci. Preprint No. 1203)

Mathys G., Hubrig S., Landstreet J.D., Lanz T., Manfroid J., 1997, A\&AS 123, 353

Mathys G., Manfroid J., 1985, A\&AS 60, 17

Morgan W.W., 1932, ApJ 75, 46

Morrison N.D., Wolff S.C., 1971, PASP 83, 474

Muthsam H., Weiss W.W., 1978, Infrared radii of $\alpha^{2}$ CVn and $\alpha$ And. In: Weiss, W.W. \& Kreidl, T.J. (eds.) Proc. Workshop Vienna October 9-10, 1978, Ap-Stars in the Infrared, p. 37

North P., 1984, A\&AS 55, 259

North P., Adelman S.J., 1995, A\&AS 111, 41

Panov K., Schöneich W., 1975, Changes of the Periods in Ap Stars. In: Aslanov I.A. (ed.) Proc. Baku Conference, Magnetic Ap Stars, p. 16 
Pilachowski C.A., Bonsack W.K., 1975, PASP 87, 221

Pilachowski C.A., Bonsack W.K., Wolff S.C., 1974, A\&A 37, 275

Preston G.W., 1969, ApJ 158, 243

Preston G.W., 1970, ApJ 160, 10596

Preston G.W., 1972, ApJ 175, 456

Preston G.W., 1974, ARA\&A 12, 257

Preston G.W., Pyper D.M., 1965, ApJ 142, 983

Przybylski A., 1961, Nat 189, 739

Przybylski A., 1963, Acta Astron. 13, 217

Przybylski A., 1966, Nat 210, 20

Przybylski A., 1977a, MNRAS 178, 735

Przybylski A., 1977b, Proc. Astr. Soc. Australia 3, 143

Rakosch K.D., 1962, Lowell Obs. Bull. 5, 227

Rakosch K.D., Fiedler W., 1978, A\&AS 31, 83

Renson P., Maitzen H.M., 1978, A\&A 65, 299

Renson P., Manfroid J., 1977, Inf. Bull. Var. Stars 1280

Renson P., Manfroid J., 1978, A\&AS 34, 445

Renson P., Manfroid J., 1980, Inf. Bull. Var. Stars 1755

Renson P., Gerbaldi M., Catalano F.A., 1991, A\&AS 89, 429

Renson P., Manfroid J., Heck A., 1976, A\&A, 23, 413

Renson P., Manfroid J., Heck A., Mathys G., 1984, A\&A 131, 63

Ryabchicova T.A., Pavlova V.M., Davydova E.S., Piskunov
N.E., 1996, Astron. Lett. 22, 822

Schöneich W., Hildebrandt G., Furtig W., 1976, Astron. Nachr. 297, 39

Shallis M.J., Blackwell D.E., 1979, A\&A 79, 48

Stepien K., 1968, ApJ 154, 945

Stibbs D.W.N., 1950, MNRAS 110, 395

Thompson I.B., 1983, MNRAS 205, 43P

van den Heuvel E.P.J., 1971, A\&A 11, 461

van Genderen A.M., 1971, A\&A 14, 48

van Dijk W., Kerssies A. Hammerschlag-Hensberge G., Wesselius P.R., 1978, A\&A 66, 187

Wegner G., 1979, Lect. Notes Phys. 125, 467

Wegner G., Petford A.D., 1974, MNRAS 168, 557

Wegner G., Cummings D.J., Byrne P.B., Stickland D.J., 1983, ApJ 272, 646

Winzer, J.E., 1974, Thesis, University of Toronto

Wolff S.C., 1974, PASP 86, 179

Wolff S.C., 1975, ApJ 202, 127

Wolff S.C., 1978, PASP 90, 412

Wolff S.C., Bonsack W.K., 1972, ApJ 176, 425

Wolff S.C., Hagen W., 1976, PASP 88, 119

Wolff S.C., Morrison N.D., 1975, PASP 87, 231

Wolff S.C., Wolff R.J., 1971, AJ 76, 422

Wolff S.C., Wolff R.J., 1972, ApJ 176, 433 